\title{
Caffeine Enhances the Calcium-Dependent Cardiac Mitochondrial Permeability Transition: Relevance for Caffeine Toxicity
}

\author{
Vilma A. Sardão, Paulo J. Oliveira, and António J. M. Moreno \\ Centro de Neurociências e Biologia Celular de Coimbra, Departamento de Zoologia, Faculdade de Ciências e Tecnologia, \\ Universidade de Coimbra, P-3004-517 Coimbra, Portugal
}

Received July 23, 2001; accepted November 19, 2001

\begin{abstract}
C affeine Enhances the $C$ alcium-D ependent $C$ ardiac Mitochondrial Permeability Transition: Relevance for Caffeine Toxicity. Sardão, V. A., Oliveira, P. J ,, and Moreno, A. J . M. (2002). Toxicol. Appl. Pharmacol. 179, 50-56.
\end{abstract}

C affeine (1,3,7-trimethylxanthine), a compound present in beverages such as tea and coffee, is known to be toxic at high concentrations. Some of the observed clinical conditions include cardiovascular disease and reproductive disorders, among others. The possible toxic effects of caffeine on heart mitochondria are still poorly understood. The influence of caffeine on the mitochondrial permeability transition has not been clarified so far. The objective of this study was to investigate whether caffeine, at toxic concentrations, had any stimulating effect on the permeability transition of heart mitochondria isolated from $\mathbf{W}$ istar rats, as well as whether it influenced mitochondrial respiratory parameters. Our results show that caffeine reduced mitochondrial ability to accumulate calcium by increasing the susceptibility of heart mitochondria to the opening of the transition pore. Caffeine not only hindered mitochondrial capacity to recover membrane potential after calcium addition but also increased the rate of calciumdependent mitochondrial swelling and calcium-induced calcium release. The increased swelling was also observed in nonenergized mitochondria. Caffeine also showed a complex array of effects on heart mitochondrial bioenergetics, as evaluated by respiratory parameter measurements. We observed an increase in state 4 respiration and a depression in state 3 respiration, although no effect was observed on succinate-sustained mitochondrial membrane potential in the absence of calcium. Our work may be relevant to cardiovascular problems linked to caffeine toxicity and also to in vitro experiences involving caffeine-induced calcium release from the sarcoplasmic reticulum and uptake by mitochondria. $\odot 2002$ Elsevier Science (USA)

Key Words: caffeine; mitochondria; permeability transition pore; calcium.

Caffeine (1,3,7-trimethylxanthine) may well be the most consumed drug in the Western society. It is present in several beverages such as coffee, tea, and carbonated drinks. Caffeine

\footnotetext{
${ }^{1}$ To whom correspondence should be addressed. Fax: 351-239-826798; E-mail: moreno@ci.uc.pt.
}

is also used for therapeutic purposes and is included in a wide variety of fixed combination prescription drugs and over-thecounter drugs. Due to increasing self-medication as well as tea and coffee overconsumption, some problems may arise from caffeine toxicity.

It is believed that most of the pharmacological effects of caffeine arise from its antagonism of the actions of adenosine at cell surface receptors, as the values for average caffeine plasma concentrations correspond well with the inhibition constant values (Benowitz, 1990). Other mechanisms may also underlie some of the pharmacological or toxicological effects of caffeine, such as the inhibition of phosphodiesterase (Daly, 1993) or the increase in cytosolic calcium by emptying intracellular stores. However, the phosphodiesterase inhibition and the increase in cytosolic calcium may not be achieved with average doses of caffeine, as higher than therapeutic concentrations (100 to $1000 \mu \mathrm{M})$ are necessary to produce those effects (as reviewed by Carrillo and Benitez, 2000). Despite this, they may be relevant in situations involving severe caffeine abuse and toxicity (Mrvos et al., 1989; Daly, 1993). Potential toxic effects of caffeine range from a moderate increase in heart rate to more severe cardiac arrhythmias. A plasma concentration of more than $1-2 \mathrm{mM}$ is known to be lethal for adult humans (Mrvos et al., 1989; Carrillo and Benitez, 2000). Although those concentrations are very hard to achieve with normal sources of caffeine, accidental or voluntary caffeine intoxication may indeed happen (Zimmerman et al., 1985; Carrillo and Benitez, 2000). Moreover, individuals with decreased caffeine elimination, such as neonates, may achieve toxic concentrations more quickly. In this particular matter, neonates appear to be particularly susceptible to caffeine intoxication, not only by having half-lives for caffeine elimination much higher than adults, but also by having a much smaller absolute volume of distribution (Anderson et al., 1999; Carrillo and Benitez, 2000).

The possible direct caffeine toxic effects on cardiac mitochondria have received little attention until now, despite many studies in whole cells concerning the cross-talk between the endoplasmic reticulum and mitochondria after caffeine addition. Nevertheless, it has been shown that the effects of caf- 
feine on the myocardium included both ultrastructural and functional abnormalities in cardiac mitochondria of embryonic chicks (Bruyere et al., 1988) and cellular and mitochondrial disruption in the hearts of newborn rats injected with that drug (Wink et al., 1999). In the late 1970s, a study by Acosta and Anuforo (1977) described significant cytotoxicity in cultured heart cells, which included labilization of mitochondrial membranes, by very high (and nonphysiologic) caffeine concentrations $(5-20 \mathrm{mM})$. Even higher concentrations of caffeine (45-60 $\mathrm{mM})$ were shown to reduce mitochondrial calcium accumulation (De la Cruz et al., 1988). However, neither was the effect on mitochondrial transmembrane electric potential $(\Delta \Psi)^{2}$ shown nor was a proposed mechanism suggested.

The possible interaction with the widely studied calciumdependent mitochondrial permeability transition (MPT), caused by the opening of the so-called mitochondrial permeability transition pores (MPTP), would be a possibility for the decreased calcium uptake.

The MPTP is a proteinaceous complex formed when mitochondria are challenged with increasing amounts of calcium and oxidative stress (Hunter et al., 1976; Kowaltowski et al., 1996). The MPTP complex is considered to be composed of several proteins of the outer and inner mitochondrial membranes, namely the adenine nucleotide translocator, the voltage-dependent anion channel, and the matrix protein cyclophilin, among others (for review see Crompton, 1999). The MPTP may either act in a low conductance form (proposed to be the physiological form), in which it becomes permeable to protons and small ions (Ichas et al., 1997; Ichas and Mazat, 1998), or in a high conductance form, in which high amplitude mitochondrial swelling occurs, normally representing irreversible injury to mitochondria. The MPT is a widely studied phenomenon in mitochondrial bioenergetics. It is believed to participate in many cellular pathological events (Lemasters et al., 1997; Lemasters, 1998), including the proapoptotic release of cytochrome c (Zamzami et al., 1996; Hirsch et al., 1998; Skulachev, 2000).

In this work we analyzed the effects of caffeine on the cardiac calcium-dependent mitochondrial permeability transition and on mitochondrial respiratory parameters. Cardiovascular abnormalities are normally associated with caffeine toxicity, so we used heart mitochondria as a model to test the effects of caffeine in doses relevant to toxic studies (1-2 mM). As referred, individuals with reduced caffeine elimination or metabolization may achieve such concentrations.

We concluded that caffeine stimulated the opening of the mitochondrial permeability transition pore and had a very complex effect on mitochondrial respiratory parameters. Our results may be relevant for a better understanding of severe cardiovascular problems associated with caffeine toxicity.

\footnotetext{
${ }^{2}$ Abbreviations used: $\Delta \Psi$, mitochondrial electric potential; MPT, mitochondrial permeability transition; MPTP, mitochondrial permeability transition pore; RCR, respiratory control ratio; $\mathrm{TPP}^{+}$, tetraphenylphosphonium cation.
}

\section{MATERIALS AND METHODS}

Materials. All compounds, including caffeine, were purchased from Sigma Chemical Co. (St. Louis, MO). Calcium Green 5N was obtained from Molecular Probes (Eugene, OR). Caffeine was freshly prepared in warm water.

Animals. Male Wistar rats $(250-300 \mathrm{~g})$, housed at $22 \pm 2^{\circ} \mathrm{C}$ under artificial light for 12-h light/day cycle and with access to water and food ad libitum, were used throughout the experiments. The research procedure was carried out in accordance with the European Requirement for Vertebrate Animal Research.

Isolation of mitochondria from rat heart. Rat heart mitochondria from male Wistar rats (250-300 g) were prepared as previously described (Oliveira et al., 2000). Mitochondrial protein content was determined by the biuret method, calibrated with bovine serum albumin.

Measurement of mitochondrial transmembrane potential. The mitochondrial transmembrane potential was estimated with a tetraphenylphosphonium $\left(\mathrm{TPP}^{+}\right)$electrode according to Kamo et al. (1979) without correction for the "passive" binding contribution of $\mathrm{TPP}^{+}$to the mitochondrial membranes, because the purpose of the experiments was to show relative changes in the potential rather than absolute values. A matrix volume of $1.1 \mu \mathrm{l} / \mathrm{mg}$ protein was assumed. Reactions were carried out, at $25^{\circ} \mathrm{C}$, in $2 \mathrm{ml}$ of the standard media $(200 \mathrm{mM}$ sucrose, $10 \mathrm{mM}$ Tris-MOPS, $10 \mu \mathrm{M}$ EGTA, $5 \mathrm{mM} \mathrm{KH}_{2} \mathrm{PO}_{4}$ ) supplemented with $3 \mu \mathrm{M} \mathrm{TPP}^{+}, 0.5 \mu \mathrm{g}$ oligomycin, and $1 \mathrm{mg}$ of mitochondria. Energized mitochondria were obtained with $8 \mathrm{mM}$ succinate (plus $4 \mu \mathrm{M}$ rotenone). The basal line was calibrated with valinomycin. Caffeine was preincubated with the mitochondrial suspension for 3 min before mitochondrial energization.

Mitochondrial oxygen consumption. Oxygen consumption of isolated heart mitochondria was monitored polarographically with a Clark oxygen electrode connected to a suitable recorder in a 1-ml thermostated, waterjacketed, closed chamber with magnetic stirring, at $25^{\circ} \mathrm{C}$. The reaction media was composed of $200 \mathrm{mM}$ sucrose, $10 \mathrm{mM}$ Tris, $10 \mu \mathrm{M}$ EGTA, and $5 \mathrm{mM}$ $\mathrm{KH}_{2} \mathrm{PO}_{4}$. Mitochondria were suspended at a concentration of $0.5 \mathrm{mg} / \mathrm{ml}$ in the respiratory medium. State 4 respiration was measured in the presence of $8 \mathrm{mM}$ succinate (plus $4 \mu \mathrm{M}$ rotenone). One hundred nanomoles of ADP, $2 \mu \mathrm{g}$ oligomycin, and $1 \mu \mathrm{M}$ FCCP were added to induce, respectively, state 3 respiration, state 4 respiration without the contribution of passive proton conductance through the ATP synthase, and uncoupled respiration. Caffeine $(0.5-2 \mathrm{mM})$ was preincubated with mitochondria in the reaction medium for 3 min. RCR was calculated as the ratio between state 3 and state 4 respiration.

Determination of mitochondrial swelling. The changes in mitochondrial volume were followed by monitoring the classic decrease in absorbance at 540 $\mathrm{nm}$ with a Jasco V-560 spectrophotometer. The assays were performed in $2 \mathrm{ml}$ of standard media with $4 \mu \mathrm{M}$ rotenone, $0.5 \mu \mathrm{g}$ oligomycin, $8 \mathrm{mM}$ succinate, and $1 \mathrm{mg}$ protein. Caffeine was preincubated with the mitochondrial preparation for $3 \mathrm{~min}$ at $25^{\circ} \mathrm{C}$. For assays involving deenergized mitochondria, the standard media was supplemented with $4 \mu \mathrm{M}$ rotenone, $1 \mu \mathrm{M}$ FCCP, $1 \mu \mathrm{M}$ A23187, and $0.5 \mu \mathrm{g}$ oligomycin.

Calcium accumulation by heart mitochondria. Extramitochondrial free $\mathrm{Ca}^{2+}$ was measured with the hexapotassium salt of the fluorescence probe Calcium Green 5N (Rajdev and Reynolds, 1993). Heart mitochondria (0.3 mg) were resuspended in $2 \mathrm{ml}$ of buffer containing $200 \mathrm{mM}$ sucrose, $10 \mathrm{mM}$ Tris,

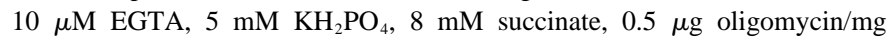
protein, and $4 \mu \mathrm{M}$ rotenone. Free extramitochondrial $\mathrm{Ca}^{2+}$ was monitored with $100 \mathrm{nM}$ Calcium Green $5 \mathrm{~N}$. Fluorescence was recorded continuously in a water-jacketed cuvette holder at $25^{\circ} \mathrm{C}$ using a Perkin-Elmer LS-50B fluorescence spectrometer with excitation and emission wavelengths of 506 and 531 $\mathrm{nm}$, respectively. At the end of each experiment, a calibration was performed calculating the maximum and minimum fluorescence signal, with an excess of calcium and EGTA, respectively. The dissociation constant used for the complex $\mathrm{Ca}^{2+}$ probe was $4 \mu \mathrm{M}$.

Statistical analysis. Values are presented as means \pm SEM and an unpaired Student's $t$ test was performed to compare values. A value of $p<0.05$ was considered significant. 


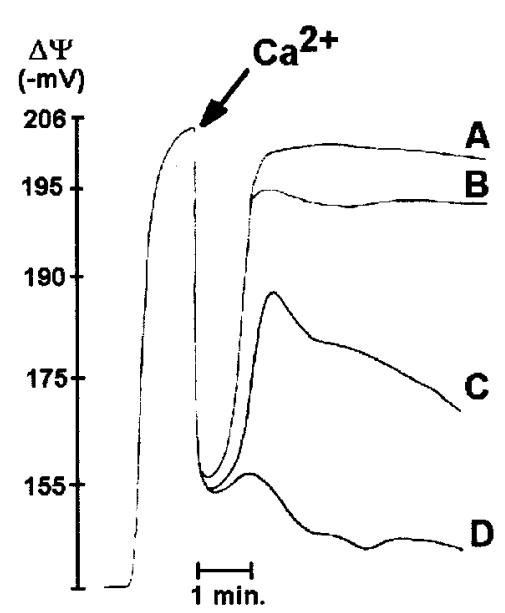

FIG. 1. Measurement of the fluctuations of mitochondrial $\Delta \Psi$ associated with calcium uptake in heart mitochondria in the presence and absence of caffeine. Incubation procedures were as described under Materials and Methods. Mitochondrial $\Delta \Psi$ was measured with a $\mathrm{TPP}^{+}$-selective electrode, without correction for $\mathrm{TPP}^{+}$passive binding to mitochondrial membranes. A calcium pulse of $400 \mu \mathrm{M}$ was added to the mitochondrial suspension (0.5 $\mathrm{mg} / \mathrm{ml}$ ). Curve B represents the control situation in the absence of caffeine. Caffeine (1 mM, curve C; $2 \mathrm{mM}$ curve D) was preincubated with mitochondria for $3 \mathrm{~min}$. Cyclosporin A $(1 \mu \mathrm{M})$, curve A, was used to inhibit the MPT induced by calcium and $2 \mathrm{mM}$ caffeine. The recording is representative of five independent preparations.

\section{RESULTS}

The effects of caffeine (1-2 mM) on the mitochondrial $\Delta \psi$ after calcium addition in the presence of phosphate can be seen in Fig. 1. The addition of $400 \mu \mathrm{M}$ calcium to succinateenergized heart mitochondria caused a sudden drop of the $\Delta \psi$ value, followed by a repolarization to a lower value of $\Delta \Psi$ after complete calcium accumulation. The same calcium pulse in the presence of caffeine (1 and $2 \mathrm{mM})$ caused a more extensive depolarization with a marked incapacity to regain the normal $\Delta \psi$ values, although we observed an almost complete repolarization of the $\Delta \Psi$ value after the addition of $2 \mathrm{mM}$ EGTA (not shown). The inhibitory effect of cyclosporin A in the presence of $2 \mathrm{mM}$ caffeine showed that the drop in the $\Delta \psi$ values was due to the stimulatory activation of the MPTP by caffeine, as cyclosporin A is considered a specific inhibitor of the pore (Broekemeier et al., 1989). ADP (1 mM) also avoided the $\Delta \Psi$ loss induced by calcium and caffeine (data not shown). We also analyzed the changes of mitochondrial oxygen consumption after the addition of calcium. Figure 2 shows that the calcium-stimulated respiration largely failed to return to normal state 4 value, showing an accelerated and mostly uncoupled oxygen consumption in the presence of $2 \mathrm{mM}$ caffeine, which was also reversed by cyclosporin A (data not shown). This was in opposition to control experiments in which respiration was restored to values close to state 4 . Caffeine by itself neither induced any $\Delta \psi$ loss nor displayed the same effect when added after the calcium pulse (data not shown). It is noticeable that caffeine, in the doses tested and in the absence

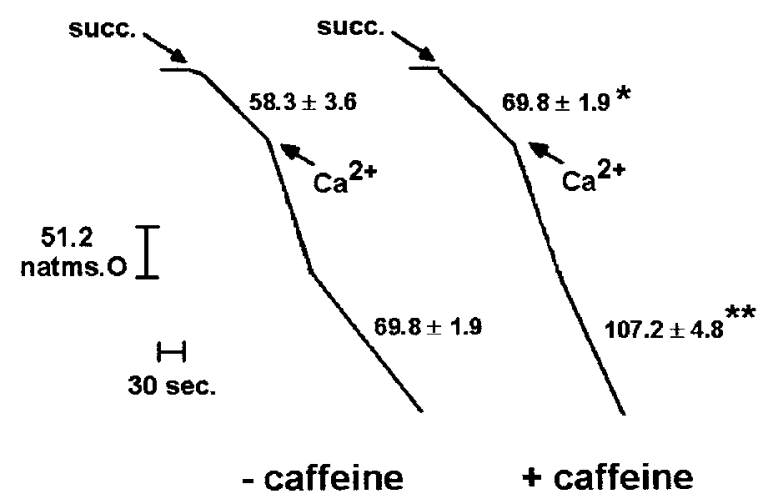

FIG. 2. Oxygen consumption by heart mitochondria monitored polarographically with a Clark oxygen electrode in a 1-ml thermostated waterjacketed closed chamber with magnetic stirring, at $25^{\circ} \mathrm{C}$. Heart mitochondria $(0.5 \mathrm{mg} / \mathrm{ml})$ were incubated in the standard reaction media as described. A calcium pulse of $400 \mu \mathrm{M}$ was added to mitochondria to observe the action of caffeine ( $2 \mathrm{mM}$, with a $3 \mathrm{~min}$ preincubation) in the recovery to state 4 respiration after calcium accumulation. Ordinates represent the nanoatoms of oxygen consumed per minute and per milligram of protein. The values on the right side of the curves represent oxygen consumption before (state 4 ) and after calcium accumulation (mean $\pm \mathrm{SEM}$ ). The recording is representative of four independent preparations. ${ }^{*} p<0.05$ vs state 4 without caffeine; ${ }^{* *} p<0.005$ vs state 4 with caffeine.

of calcium, did not hinder mitochondrial $\Delta \Psi$ generation after succinate addition.

Another hallmark of the MPT is the high-amplitude swelling induced by calcium in the presence of phosphate. In our assays, (Fig. 3) the addition of $300 \mu \mathrm{M}$ calcium caused a biphasic reduction of the mitochondrial suspension absorbance, typical of the mitochondrial swelling induced by the opening of the MPTP in heart mitochondria. Again, caffeine (1-3 mM) stimulated the rate at which mitochondria swelled in a cyclosporin

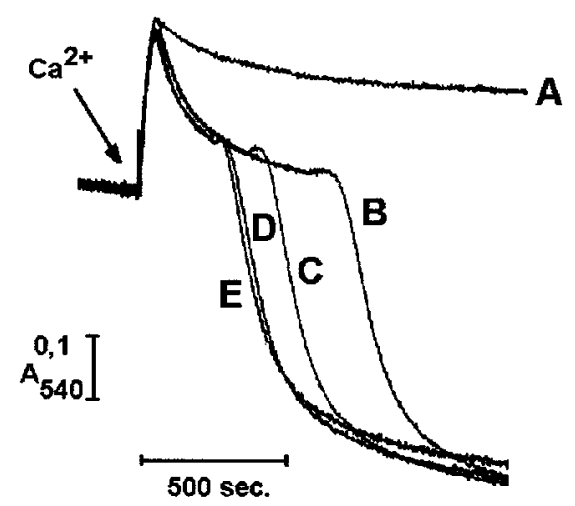

FIG. 3. Measurement of mitochondrial swelling upon induction of the MPT. The absorbance of mitochondrial suspension was followed at $540 \mathrm{~nm}$. Mitochondria $(0.5 \mathrm{mg} / \mathrm{ml})$ were incubated in the standard reaction media as described. A calcium pulse of $300 \mu \mathrm{M}$ was added to attain the cyclosporin A-sensitive swelling. Caffeine was preincubated with mitochondria for $3 \mathrm{~min}$ before calcium addition. (A) $2 \mathrm{mM}$ caffeine + cyclosporin A; (B) control curve; (C) $+1 \mathrm{mM}$ caffeine; (D) $+2 \mathrm{mM}$ caffeine; and (E) $+3 \mathrm{mM}$ caffeine. The recording is representative of five independent preparations. 


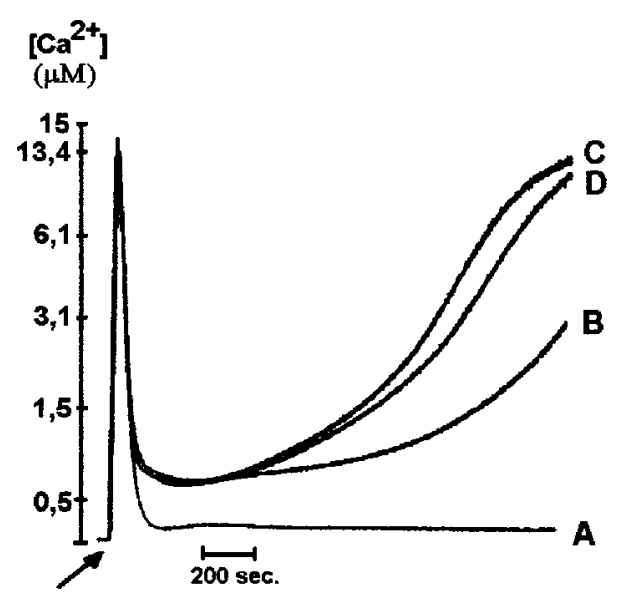

FIG . 4. Movements of extramitochondrial calcium measured with a calcium-sensitive fluorescent probe, Calcium Green $5 \mathrm{~N}$. Mitochondria $(0.3 \mathrm{mg} /$ $\mathrm{ml}$ ) were incubated in the reaction media containing $100 \mathrm{nM}$ Calcium Green $5 \mathrm{~N}$. A calcium pulse ( $15 \mu \mathrm{M}$; arrow) was added to the mitochondrial suspension and the fluorescence signal was measured. Fluorescence was recorded continuously in a water-jacketed cuvette holder at $25^{\circ} \mathrm{C}$ using a Perkin-Elmer LS-50B fluorescence spectrometer with excitation and emission wavelengths of 506 and $531 \mathrm{~nm}$, respectively. The signal calibration was performed as described. (A) $+1 \mu \mathrm{M}$ cyclosporin $\mathrm{A}+1 \mathrm{mM}$ caffeine; (B) control curve; (C) $+1 \mathrm{mM}$ caffeine; and (D) $+0.5 \mathrm{mM}$ caffeine. The recording is representative of five independent preparations.

A-sensitive manner. Caffeine, when added after calcium, did not induce mitochondrial swelling (data not shown). Caffeine (1-2 mM) caused only a nonsignificant slight increase in the rate of intramitochondrial pyridine nucleotides loss (not shown). Nevertheless, in the presence of cyclosporin A to avoid artifacts secondary to mitochondrial swelling, $2 \mathrm{mM}$ caffeine and calcium did not produce the decrease in the levels of NAD(P)H (not shown).

This caffeine-stimulated permeability transition was further studied by monitoring the calcium-buffering capacity of isolated heart mitochondria. As expected, the presence of caffeine restrained the capacity of mitochondria to buffer an added pulse of calcium. In the presence of this drug, mitochondria released the accumulated calcium much faster than in its absence, again, in a cyclosporin A-sensitive manner (Fig. 4).

We further evaluated the effects of the tested caffeine concentrations on the mitochondrial respiratory chain. In order to pursue this objective, we determined the mitochondrial oxygen consumption in the presence and the absence of several caffeine concentrations. Some respiratory parameters were evaluated, as seen in Fig. 5 (top and bottom), including the effects on state 4 , state 3 , and uncoupled respiration. We obtained a significant stimulatory effect of caffeine on state 4 respiration. This stimulation was not so visible in the presence of oligomycin. State 3 respiration was also inhibited by caffeine, which explains the significant reduction of the RCR values. Additionally, uncoupled respiration was also inhibited by caffeine in the range of concentrations tested. Nevertheless, the ADP/O (num- ber of nanomoles ADP phosphorylated by nanoatoms oxygen consumed) value was not significantly affected (not shown).

Due to the complexity of caffeine effects on mitochondrial respiratory parameters, it was necessary to ensure that any effects on pore opening were not secondary to energization or electrophoretic calcium transport into mitochondria. Therefore, we used deenergized mitochondria to observe the effects of caffeine in the mitochondrial swelling after addition of a calcium pulse in the presence of A23187, a calcium ionophore. Figure 6 shows that caffeine $(1-2 \mathrm{mM})$ stimulated the rate and extension of mitochondrial swelling after calcium addition. A very important observation was that cyclosporin A inhibited the observed swelling to the same extent either in the presence of $2 \mathrm{mM}$ caffeine or in its absence. Caffeine did not have any effect without calcium addition.
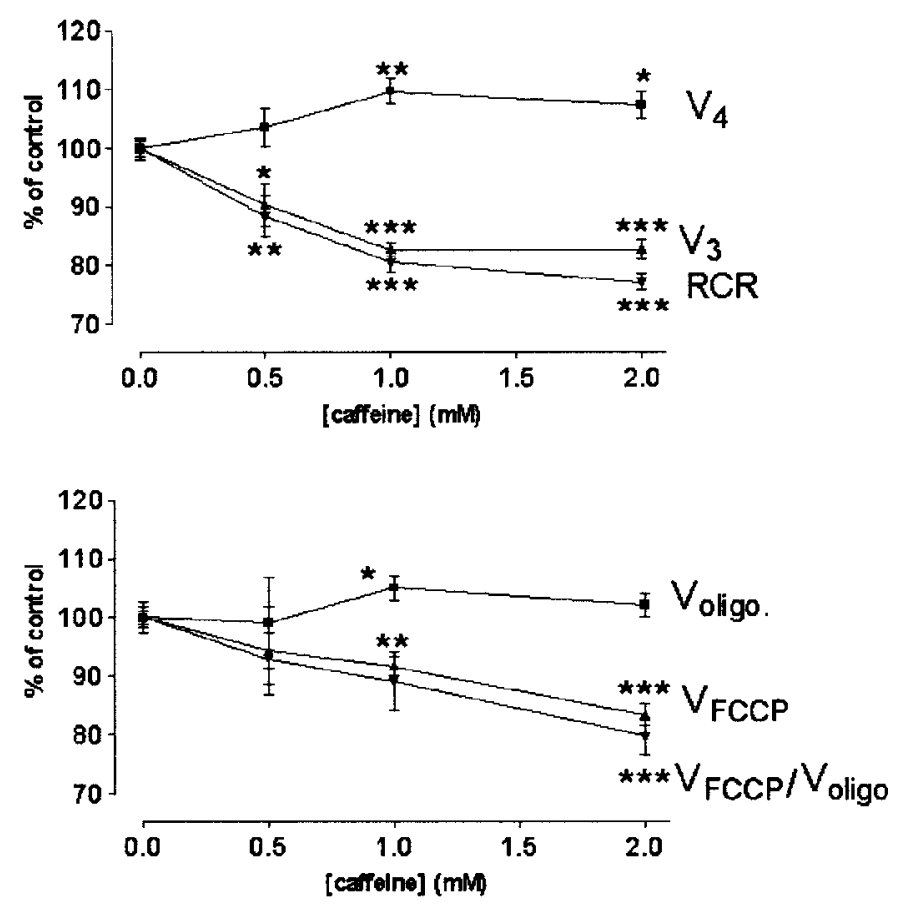

FIG. 5. Caffeine effects in mitochondrial respiratory parameters. Oxygen consumption of isolated heart mitochondria was monitored polarographically with a Clark oxygen electrode connected to a suitable recorder in a 1-ml thermostated water-jacketed closed chamber with magnetic stirring, at $25^{\circ} \mathrm{C}$. Mitochondria were suspended at a concentration of $0.5 \mathrm{mg} / \mathrm{ml}$ in the respiratory medium. State $4\left(\mathrm{~V}_{4}\right)$ respiration was measured in the presence of $8 \mathrm{mM}$ succinate (plus $4 \mu \mathrm{M}$ rotenone). ADP (100 nmol), oligomycin $(2 \mu \mathrm{g})$, and FCCP $(1 \mu \mathrm{M})$ were added to induce, respectively, state $3\left(\mathrm{~V}_{3}\right)$ respiration, state 4 respiration without the contribution of passive proton conductance through the ATP synthase, and uncoupled respiration. Caffeine $(0.5-2 \mathrm{mM})$ was preincubated with mitochondria in the reaction medium for 3 min. RCR was calculated as the ratio between state 3 and state 4 respiration. Also shown is the variation on oxygen consumption after oligomycin $\left(\mathrm{V}_{\text {olig }}\right)$ and FCCP addition $\left(\mathrm{V}_{\mathrm{FCCP}}\right)$ and on the ratio $\mathrm{V}_{\mathrm{FCCP}} / \mathrm{V}_{\text {oligo }}$. All values represent the average $\pm \mathrm{SEM}$ and were calculated as a percentage of control values (absence of caffeine). Statistical analysis was conducted using the unpaired $t$ test. ${ }^{*} p<0.05$ vs control; ** $p<0.01$ vs control, $* * * p<0.005$ vs control. 


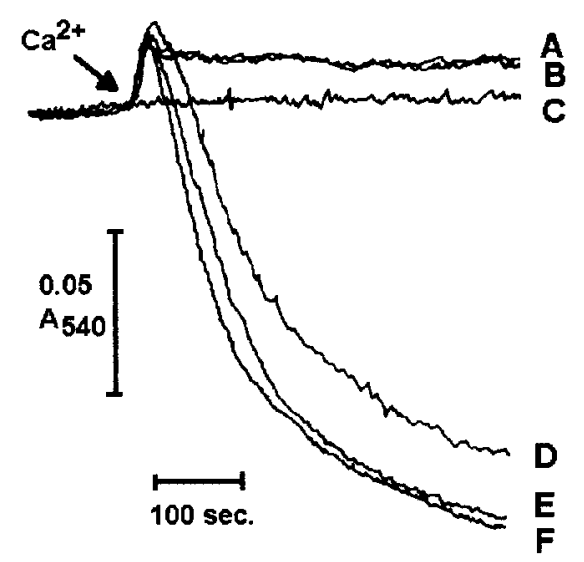

FIG. 6. Measurement of mitochondrial swelling upon induction of the MPT in denergized mitochondria. The absorbance of mitochondrial suspension was followed at $540 \mathrm{~nm}$. Mitochondria $(0.5 \mathrm{mg} / \mathrm{ml})$ were incubated in the standard reaction media supplemented with $0.5 \mu \mathrm{g}$ oligomycin, $1 \mu \mathrm{M}$ FCCP, and $1 \mu \mathrm{M}$ A23187. A calcium pulse of $400 \mu \mathrm{M}$ was added in order to attain the cyclosporin A-sensitive swelling. Caffeine was preincubated with mitochondria for $3 \mathrm{~min}$ before calcium addition. (A) $+2 \mathrm{mM}$ caffeine $+1 \mu \mathrm{M}$ cyclosporin-A; (B) $+1 \mu \mathrm{M}$ cyclosporin A; (C) $2 \mathrm{mM}$ caffeine without calcium addition; (D) control curve (calcium alone); (E) $+1 \mathrm{mM}$ caffeine; and (F) $+2 \mathrm{mM}$ caffeine. The recording is representative of three independent preparations.

\section{DISCUSSION}

Caffeine is a common component in many beverages and medications. Its abusive consumption may lead to some cardiac disorders that may prove fatal.

The effects of caffeine in mitochondrial calcium handling have not been fully analyzed so far. Bearing this in mind, we investigated how caffeine affects the calcium-dependent mitochondrial permeability transition. To study the $\Delta \Psi$ loss associated to the MPT, we used a $\mathrm{TPP}^{+}$-selective electrode, which allows a continuous monitoring of the mitochondrial $\Delta \Psi$ fluctuations. As seen in Fig. 1, the addition of a calcium pulse to heart mitochondria caused a depolarization followed by membrane repolarization. In the presence of caffeine (1 and $2 \mathrm{mM}$, preincubated for $3 \mathrm{~min}$ ), heart mitochondria were unable to return to the normal $\Delta \psi$ values, a phenomenon that was reverted by EGTA (not shown). The stronger effect was observed for $2 \mathrm{mM}$ caffeine, where no repolarization was observed. This $\Delta \psi$ loss was prevented by cyclosporin A, a known inhibitor of the MPTP (Broekemeier et al., 1989) and $1 \mathrm{mM}$ ADP. Therefore, we conclude that the $\Delta \psi$ loss was directly related to the stimulated opening of the MPTP by caffeine. We also observed that this effect only occurred when calcium was added after caffeine preincubation (data not shown) and that the ion was needed for the effect of caffeine (not shown). Further work is underway in our laboratory to explain these results. We can speculate that caffeine may help expose some calcium-binding sites in the pore complex, decreasing the matricial calcium threshold for pore opening. It is reasonable to think that, after complete calcium accumulation inside mitochondria, those sites are no longer accessible to caffeine.
Caffeine induction of the MPTP was also evident following oxygen consumption by the heart mitochondrial suspension (Fig. 2). As pictured, in the presence of $2 \mathrm{mM}$ caffeine and after a calcium pulse, the mitochondrial respiratory rate did not return to normal state 4 values, showing a mitochondrialuncoupled state. This differed from the control assay, in which respiration returned to state 4 values following complete calcium accumulation by mitochondria. The presence of cyclosporin A inhibited the uncoupled state caused by caffeine (not shown). The differences in oxygen consumption after calcium accumulation in both experiments allowed us to conclude that a larger set of mitochondria in the population suffered the MPTP in the presence of caffeine than in control experiments.

Mitochondrial swelling (Fig. 3) and the calcium-buffering capacity (Fig. 4) were also studied. Again, in the presence of caffeine, the rate at which mitochondria swelled and released the accumulated calcium was stimulated. Both effects were cyclosporin A sensitive and revealed that caffeine reduced the mitochondrial capacity to accumulate calcium. Caffeine only displayed the observed effect when it was added before calcium, as already described for the $\Delta \psi$ assays. Concerning mitochondrial swelling, the profile of the curves was typical for heart mitochondria isolated with our current procedure. As described elsewhere, the heart possesses two mitochondrial populations, one located beneath the sarcolemma and another one located between the myofibrils, which have different properties concerning calcium uptake and accumulation capacity (Palmer et al., 1977, 1986). This may lead the final swelling curve to display a biphasic form (Fig. 3). Furthermore, the large increase in absorbance observed after calcium addition can be explained as calcium precipitation in the mitochondrial matrix, as previously described (Andreyev et al., 1998), due to the high calcium concentration employed.

Respiratory parameters in the presence and the absence of caffeine were evaluated in order to identify some direct effects in the respiratory chain. In succinate-energized heart mitochondria, we evaluated the caffeine-induced alteration of mitochondrial respiratory states. As seen in Fig. 5 and previously described under Results, caffeine increased the respiration in state 4 , not only in the absence of oligomycin $\left(\mathrm{V}_{4}\right)$, but also upon its addition $\left(\mathrm{V}_{\text {oligo }}\right)$. This result could suggest an uncoupler or decoupler action. As caffeine did not decrease the mitochondrial $\Delta \psi$ for the tested concentrations (see Fig. 1, before calcium addition), we concluded that an uncoupler mode of action is unlikely to be responsible for the observable respiratory stimulation. This was supported by the fact that, in the presence of oligomycin (thus excluding passive proton leak through the ATP synthase), the effect was less enhanced, especially for $2 \mathrm{mM}$ caffeine. This behavior led us to conclude that a decoupler mode of action might have been responsible for the stimulation of state 4 respiration (Murphy, 1989), since the mitochondrial $\Delta \Psi$ was not affected, even in the presence of $2 \mathrm{mM}$ caffeine. Our results showed that caffeine also affected state 3 respiration. As a consequence of both state 4 and state 
3 alterations, the RCR value suffered a significant decrease. Nevertheless, the P/O index was not significantly altered (data not shown). Uncoupled respiration with FCCP was also decreased by caffeine. This suggested that caffeine also affected the respiratory chain, hindering the normal electron flux, during conditions in which mitochondrial respiration is maximally stimulated. The idea that caffeine may act on the adenine nucleotide translocator should not be excluded, as it would agree with some of our data, including the stimulatory effect in the MPT or the decreased state 3 respiration. It has been proposed that the adenine nucleotide translocator is, in fact, one of components of the MPTP complex (Vieira et al., 2000). Further work will be carried out in our laboratory to clarify the precise mechanisms of action of caffeine in heart mitochondria.

Due to the apparent complexity of caffeine effects in bioenergetic parameters in heart mitochondria, it was pertinent to see if caffeine still displayed the same stimulatory effect in the MPT in deenergized mitochondria. Figure 6 shows that caffeine also enhanced the rate of mitochondrial swelling, in a cyclosporin A-sensitive manner, showing that the results were not due to nonspecific destruction of mitochondria but to a possible direct effect on any of the pore components. The study revealed that caffeine hindered the capacity of heart mitochondria to buffer the external calcium, which may reveal itself damaging to the role of mitochondria in the cell. Sustained enhancement of the MPTP by caffeine may cause a decrease in mitochondrial ATP production, large amplitude swelling with concomitant mitochondrial structural damage, as well as cell death (Crompton, 1999). Those events will ultimately contribute to the heart failure normally associated with severe caffeine intoxication (Mrvos et al., 1989), as the cardiac muscle would suffer from a decreased supply of mitochondrial-derived ATP and a diminished mitochondrial capacity to help maintain cytosolic calcium homeostasis.

The concentrations used in our study are well above the average concentrations found in the plasma after typical dietary supplementation. Nevertheless, it is known that during heavy medication or accidental or suicidal ingestion, caffeine concentration in the plasma may reach concentrations well above $1 \mathrm{mM}$ (Mrvos et al., 1989; Carrillo and Benitez, 2000).

The studied caffeine concentrations are within the range used by many authors to extrude calcium from the sarcoplasmic reticulum $(1-20 \mathrm{mM})$ in in vitro experiments involving cardiac cells. Facing this, we consider that caution should be used to verify whether caffeine is not limiting mitochondrial capacity to buffer the released calcium. As mitochondria were described to be located in close proximity of the sarcoplasmic reticulum (Mannella, 2000), the combination of caffeine and calcium may stimulate the induction of the MPTP in mitochondria, thus leading to an underestimation of the calcium taken up by mitochondria. Also, attention should be given to the recently discovered ryanodine receptors in rat heart mitochondria
(Beutner et al., 2001). At the present time, we are unable to say if these receptors may have some responsibility for the observed effect of caffeine. Another possibility to explore in the future is to verify whether caffeine metabolites present the same kind of effect in isolated rat heart mitochondria.

\section{ACKNOWLEDGMENTS}

Paulo J. Oliveira is supported by a Ph.D grant from the Portuguese Foundation for Science and Technology (PRAXIS XXI/BD/21494/99). The authors thank Andreia Serra, Manuela Ferreira, and Sara Geraldo for some preliminary studies. We also thank Jim Bjork and Tim O'Brien for reviewing the English.

\section{REFERENCES}

Acosta, D., and Anuforo, D. (1977). Acute mitochondrial toxicity of caffeine in cultured heart cells. Drug Chem. Toxicol. 1, 19-24.

Anderson, B. J., et al.(1999). Caffeine overdose in a premature infant: Clinical course and pharmacokinetics. Anaesth. Intensive Care 27, 307-311.

Andreyev, A. Y., et al. (1998). Cytochrome $c$ release from brain mitochondria is independent of the mitochondrial permeability transition. FEBS Lett. 439, 373-376.

Benowitz, N. L. (1990). Clinical pharmacology of caffeine. Annu. Rev. Med. 41, 277-278.

Beutner, G., et al. (2001). Identification of a ryanodine receptor in rat heart mitochondria. J. Biol. Chem. 276, 21482-21488.

Broekemeier, K. M., et al. (1989). Cyclosporin A is a potent inhibitor of the inner membrane mitochondrial transition in liver mitochondria. J. Biol. Chem. 264, 7826-7830.

Bruyere, H. J., Jr., et al. (1988). The effects of caffeine on the ultrastructure and mitochondrial function of the embryonic chick heart. J. Appl. Toxicol. 8, 9-13.

Carrillo, J. A., and Benitez, J. (2000). Clinically significant pharmacokinetic interactions between dietary caffeine and medications. Clin. Pharmacokinet. 39, 127-153.

Crompton, M. (1999). The mitochondrial permeability transition pore and its role in cell death. Biochem. J. 341, 233-249.

Daly, J. W. (1993). Mechanisms of action of caffeine. In Caffeine, Coffee, and Health (S. Garanttini, Ed.), pp. 97-150. Raven Press, New York.

De la Cruz, M. J., et al. (1988). Changes induced by caffeine, theophylline and theobromine on calcium uptake, respiration and ATP levels in rat-liver mitochondria. Comp. Biochem. Physiol. 91C, 443-447.

Hirsch, T., et al. (1998). Mitochondrial permeability transition in apoptosis and necrosis. Cell Biol. Toxicol. 14, 141-145.

Hunter, D. R., et al. (1976). Relationship between configuration, function, and permeability in calcium-treated mitochondria. J. Biol. Chem. 251, 50695077.

Ichas, F., and Mazat, J.-P. (1998). From calcium signaling to cell death: Two conformations for the mitochondrial permeability transition pore: Switching from low- to high-conductance state. Biochim. Biophys. Acta 1366, 33-50.

Ichas, F., et al. (1997). Mitochondria are excitable organelles capable of generating and conveying electrical and calcium signals. Cell 89, 11451153.

Kamo, N., et al. (1979). Membrane potential of mitochondria measured with an electrode sensitive to tetraphenyl phosphonium and relationship between proton electrochemical potential and phosphorylation potential in steady state. J. Membr. Biol. 49, 105-121. 
Kowaltowski, A. J., et al. (1996). Effect of inorganic phosphate concentration on the nature of inner mitochondrial membrane alterations mediated by $\mathrm{Ca}^{2+}$ ions. J. Biol. Chem. 271, 2929-2934.

Lemasters, J. J. (1998). The mitochondrial permeability transition: From biochemical curiosity to pathophysiological mechanisms. Gastroenterology 115, 783-786.

Lemasters, J. J., et al. (1997). The mitochondrial permeability transition in toxic, hypoxic and reperfusion injury. Mol. Cell. Biochem. 174, 159-165.

Mannella, C. A. (2000). Introduction: Our changing views of mitochondria. J. Bioenerg. Biomembr. 32, 1-4.

Mrvos, R. M. et al. (1989). Massive caffeine ingestion resulting in death. Vet. Hum. Toxicol. 31, 571-572.

Murphy, M. P. (1989). Slip and leak in mitochondrial oxidative phosphorylation. Biochim. Biophys. Acta 977, 123-141.

Oliveira, P. J., et al. (2000). Carvedilol inhibits the exogenous NADH dehydrogenase in rat heart mitochondria. Arch. Biochem. Biophys. 374, 279-285.

Palmer, J. W., et al. (1977). Biochemical properties of subsarcolemmal and interfibrillar mitochondria isolated from rat cardiac muscle. J. Biol. Chem. 252, 8731-8739.
Palmer, J. W., et al. (1986). Heterogeneous response of subsarcolemmal heart mitochondria to calcium. Am. J. Physiol. 19, H741-H748.

Rajdev, S., and Reynolds, I. J. (1993). Calcium Green-5N, a novel fluorescent probe for monitoring high intracellular free $\mathrm{Ca}^{2+}$ concentrations associated with glutamate excitotoxicity in cultured rat brain neurons. Neurosci. Lett. 162, 149-152.

Skulachev, V. P., (2000). Mitochondria in the programmed death phenomena: A principle of biology: "It is better to die than to be wrong". IUBMB Life 49, 365-373.

Vieira, H. L. A., et al. (2000). Permeabilization of the mitochondrial inner membrane during apoptosis: Impact of the adenine nucleotide translocator. Cell Death Differ. 7, 1146-1154.

Wink, C. S., et al. (1999). Effects of caffeine on heart mitochondria in newborn rats. Biol. Neonate 76, 114-119.

Zamzami, N., et al. (1996). Inhibitors of permeability transition interfere with the disruption of the mitochondrial transmembrane potential during apoptosis. FEBS Lett. 384, 53-57.

Zimmerman et al. (1985). Caffeine intoxication: A near fatality. Ann. Emerg. Med. 14, 1227-1229. 